\title{
Is Marine w-3 Long Chain Polyunsaturated Fatty Acids Intake, Natural Remedial Measure for Combating Global COVID-19 Pandemic?: A Mini-Review
}

\author{
R Anandan', M Rosemol Jacob ${ }^{1,2}$, TS Fazil', CN Ravishankar', Suseela Mathew ${ }^{1 *}$ \\ ${ }^{1}$ ICAR-Central Institute of Fisheries Technology, Matsyapuri (PO), Cochin-682029, India \\ ${ }^{2}$ Department of Marine Biology, Microbiology and Biochemistry, Cochin University of Science and Technology,
} Cochin-682016, India

\begin{abstract}
*Address for Correspondence: Dr. Suseela Mathew, Principal Scientist and Head, Biochemistry and Nutrition Division, ICAR-Central Institute of Fisheries Technology, Matsyapuri (PO), Cochin-682029, India

E-mail: suseela1962@gmail.com
\end{abstract}

Received: 21 Aug 2020/ Revised: 23 Oct 2020/ Accepted: 28 Dec 2020

\begin{abstract}
COVID-19 is considered as the most public health emergency of international concern and global scientific research is on fast track to reduce the rise of epidemic curves. Marine natural bioactive molecules have been the starting point for the discovery of the most important drugs. The marine oils rich in $\omega-3$ long chain polyunsaturated fatty acids ( $\omega-3$ LCPUFAs), especially, eicosapentaenoic acid (EPA) and docosahexaenoic acid (DHA) are well known to exert promising human healthcare benefits such as cardioprotective, antilipidemic, anti-inflammatory, anticancer, antiaging, antihypertensive, antidepression and antiarthritic properties. Interestingly, the immunomodulatory and anti-inflammatory potential of $\omega-3$ LCPUFAs is capable of attenuating some of the COVID-19 mediated pathophysiological abnormalities through inhibition of proinflammatory processes. Understanding the basic mechanism of action of $\omega-3$ LCPUFAs on pathophysiological conditions associated with COVID -19 may help address the pandemic related healthcare complications. Classical mechanism of action of $\omega-3$ LCPUFAs in exhibiting anti-inflammatory properties is probably through counteraction of nuclear factor-kB activation, which is a transcription factor potentially involved in COVID-19 mediated of proinflammatory cytokine production. Also, dietary $\omega-3$ LCPUFAs intake is well known to activate generation of resolvins and protectin, which in turn can suppress COVID-19 associated inflammation responses. Considering the beneficial actions of dietary intake of $\omega-3$ LCPUFAs rich marine oils, this mini-review elaborates the physiologic and metabolic influences of $\omega$-3 LCPUFAs on COVID-19 induced pathophysiological aberrations with relevance to their biochemical and molecular functions.
\end{abstract}

Key-words: COVID-19, Docosahexaenoic acid, Eicosapentaenoic acid, w-3 PUFA, Sardinella longiceps

\section{INTRODUCTION}

COVID-19 is a new public health crisis intimidating the human population with the emergence and spread of novel coronavirus causing severe acute respiratory syndrome. Scientific investigation on pathogenic and pathophysiological processes associated with COVID-19 infection has attracted the research community to explore the scope of natural resources to counteract/ reduce the incidence of dreadful pandemic.

\footnotetext{
How to cite this article

Anandan R, Jacob MR, Fazil TS, Ravishankar CN, Mathew S. Is marine $\omega-3$ Long Chain Polyunsaturated Fatty Acids intake, Natural Remedial Measure for Combating Global COVID-19 Pandemic?: A Mini-Review. SSR Inst. Int. J. Life Sci., 2021; 7(1): 2749-2753.
}

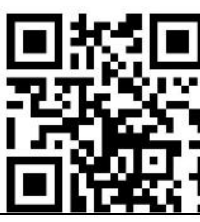

Access this article online https://iijls.com/
Clarity has not yet come whether a drug or a vaccine or food itself can be a promising candidate in addressing the physiological concerns associated with COVID-19. Globally scientific research programs are on fast track to boost both innate and acquired immunity responses among human population to fight against both the present and future virulent strains of SARS-CoV-2. Interestingly, the recent therapeutic interventions carried out worldwide with oral supplementation of $\omega-3$ LCPUFAs in controlling the incidence of COVID $-19^{[1,2]}$ has pointed out at our concept of food as medicine regaining therapeutic attention in treating diseases/disorders.

Though evolution depicts the origin of life from sea with subsequent conquering of terrestrial environment with desirable acquired characteristics, it makes us to rethink whether organisms in the land may still rely more upon 
marine environment for their healthcare purposes. It may not be a surprise if scientific research soon finds a solution from $\omega-3$ LCPUFAs rich marine oils for successful counteraction of pathophysiological conditions associated with COVID 19. Incidence of COVID-19 and associated complications are relatively low in Kerala, India, which comprises of larger number of marine fish eating population. It is high time to focus scientific research on the biochemical and molecular mechanisms of $\omega-3$ LCPUFAs concerning of the infectious processes associated with COVID -19. Comparing the levels of research on the effects of fish oil in nutrition, the scientific understanding of the dietary influence of $\omega-3$ LCPUFAs on immunological functions during viral and/or bacterial infections is relatively scanty. In this minireview, the impact of $\omega-3$ LCPUFAs on COVID-19 induced pathophysiological alterations has been discussed with relevance to their biochemical and molecular functions [3].

\section{Molecular mechanisms of action of $\omega-3$ LCPUFA against}

Covid-19- A better understanding of virus-host interactions is essential to establish the novel therapeutic applications of $\omega-3$ LCPUFAs in controlling COVID-19 pandemic. Apart from the common symptoms such as headache, cough, fever, sputum production, and diarrhoea, the disease manifestation of COVID-19 illness includes RNAaemia, acute respiratory distress syndrome, acute cardiac injury, and lung infection, which are due to inflammation related systemic and localized immune response ${ }^{[4]}$. It is not clear whether the mechanism of action of $\omega-3$ LCPUFAs on immune response is mainly attributed to inhibition of lipid microdomain clustering or stimulation of B cell populations ${ }^{[5]}$. Moreover, its role in activation of surface markers and inhibition of nuclear factor-kB induced cytokine storm cannot be overruled. It is interesting to note that the inhibitory actions of $\omega-3$ LCPUFAs on the activities of membrane-bound aminopeptidase, angiotensin-converting enzyme 2 (ACE2) ${ }^{[6]}$, which has been identified as a functional receptor for the binding of the spike protein of the corona virus to invade epithelial cells, may probably be responsible for normalizing respiratory and cardiovascular complications in COVID-19 patients ${ }^{[7]}$.

The possible shutdown actions of $\omega-3$ LCPUFAs on the alternate mechanisms of COVID-19 mediated cellular dysfunction, which include imbalanced response of $T$ helper cells and cytokine storm warrants a surprise research package for immunological scientists ${ }^{[8]}$. Also probably the $\omega-3$ LCPUFAs mediated eicosanoid production and generation of mediators like resolvins ${ }^{[9]}$ may be playing an important role in dissolving the lipophilic capsid and destroying virus. Modulation in the fatty acid composition of cell membrane of lymphocytes, sphingolipid/cholesterol-enriched lipid domains, and membrane fluidity, which play major roles in improving cell signalling, are the promising aspects of dietary influence of $\omega-3$ LCPUFAs to be addressed in detail by nutrigenomic scientific community. The $\omega-3$ LCPUFA metabolites are well known to modulate eicosanoid production, gene expression, lipid raft composition and T-cell signalling ${ }^{[10]}$ (Fig. 1).

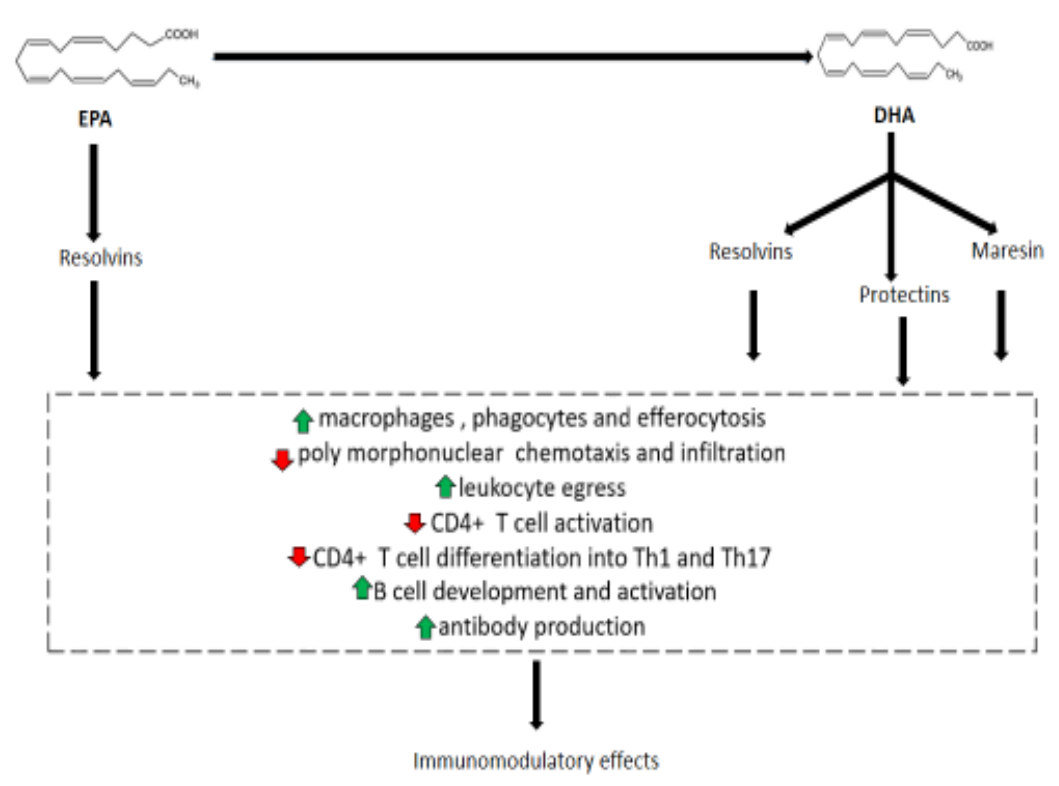

Fig. 1: Immune booster mechanism of action of EPA and DHA 
Fish oil mediated induction of TNF- $\alpha$, IL- 6 and IFN- $\gamma^{9}$ can make $B$ cells more efficient in combating virulent pathogens. Globally, the ageing associated deficiency in $\omega-3$ LCPUFAs concentrations in cellular system of brain, heart, lungs and kidney may be one of the major factors responsible for the increased mortality rate noticed in elderly population. Most importantly, the COVID-19 associated coagulopathy and thrombosis could be effectively managed with the help of anticoagulant property of $\omega-3$ LCPUFAs ${ }^{[11]}$. The anticoagulant function of $\omega-3$ PUFA is possibly related to the increased retention of uncarboxylated coagulation factors, which can be considered as an independent mechanism of action of marine $\omega-3$ LCPUFAs apart from regular hepatic synthesis of coagulation factors ${ }^{[12]}$.
Dietary intake of fish oil rich in eicosapentaenoic acid (EPA) and docosahexaenoic acid (DHA) may possibly attenuate the COVID-19 lockdown mediated sedentary life style, which caused obesity and memory decline in both young children and elderly population. The marine small pelagic Indian oil sardine, Sardinella longiceps (Fig. 2), contains rich amount of EPA, and DHA (Fig. 3), which are well known for prevention of myocardial infarction in human beings ${ }^{[13]}$. Supplementation of $\omega-3$ LCPUFAs could possibly, not only boost innate and acquired immune systems against COVID-19; but also reduce uncertainty regarding management of the complications that arise in the course of corona viral illness.

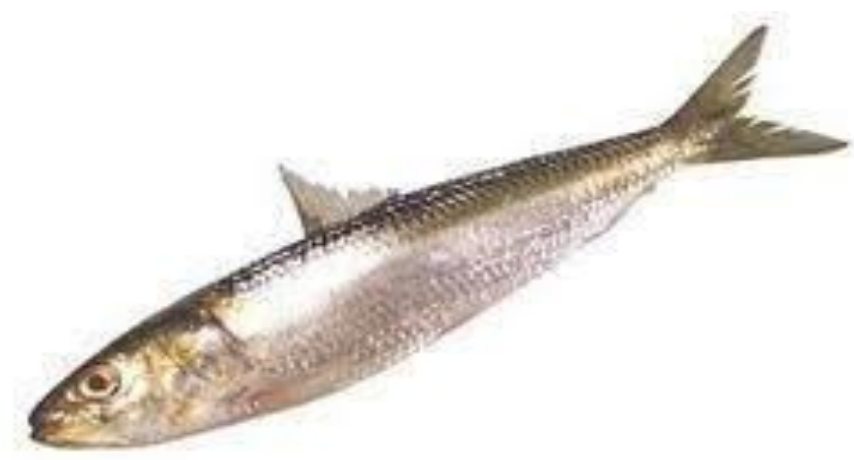

Fig. 2: Indian oil sardine (Sardinella longiceps), a marine fish rich in $\omega-3$ LCPUFAs

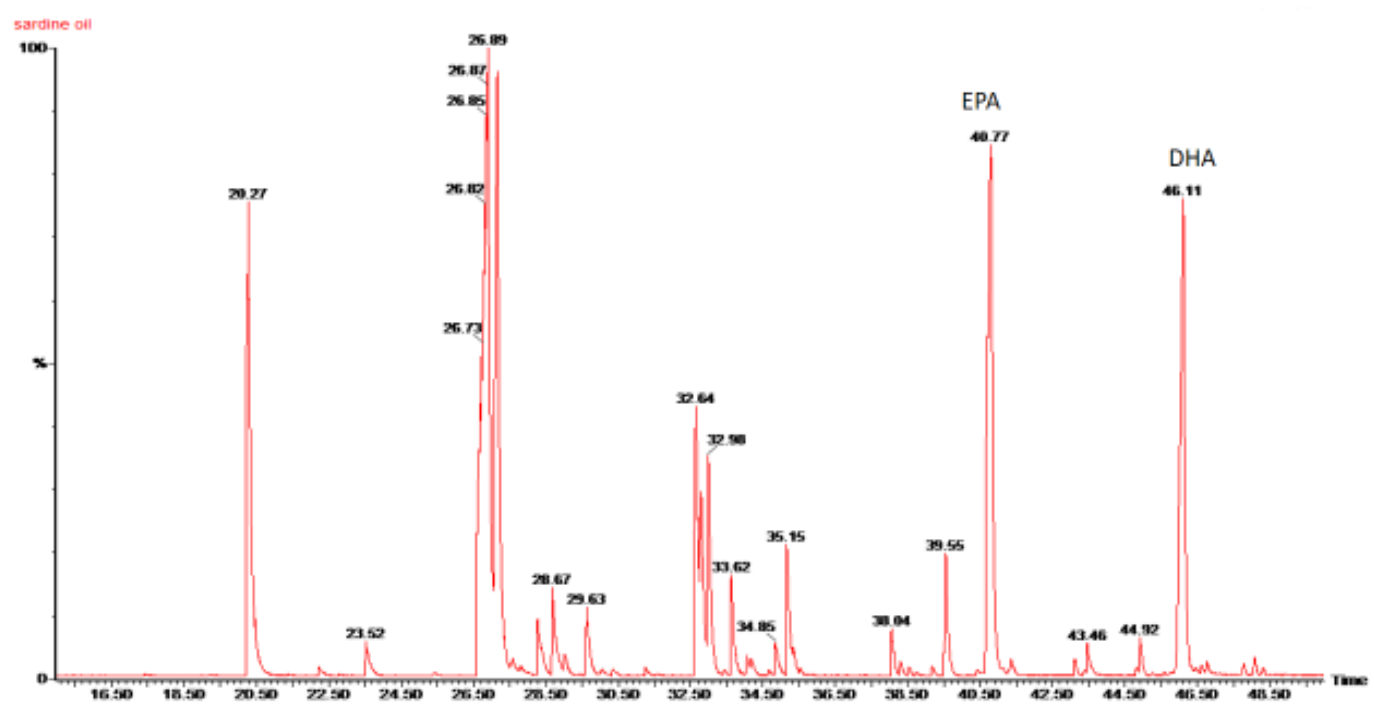

Fig. 3: Gas Chromatographic Pattern of EPA and DHA in Sardinella longiceps

\section{CONCLUSIONS}

In conclusion, the immunomodulatory and antiinflammatory potential of $\omega-3$ LCPUFAs is possibly proficient in diminishing COVID-19 associated pathophysiological irregularities through inhibition of pro-inflammatory processes.

The healthcare complications related to the pandemic can be reduced, at least in part, through dietary intake of $\omega-3$ LCPUFAs. The possible mechanism of action of 
dietary $\omega$-3 LCPUFAs is related to their ability to generate resolvins and protectin, which in turn can subdue COVID19 associated inflammation responses. Natural biomolecules have been the starting point for the discovery of most important drugs. Also finding out the possible research gaps on the basic mechanism of action of $\omega-3$ LCPUFAs on pathophysiological conditions associated with COVID -19 may pave way for more focussed research addressing the healthcare functions and therapeutic applications of fish oil.

Since India is one of the largest fish oil producers and exporters in the world, it will be no wonder, if marine fish oils find a place to boost the economy of India, the country which is considered as Global Pharmaceutical Hub. Though the nutraceutical and therapeutic values of fish oil are promising, there is an urgent need to carry out extensive research to confirm the exact molecular mechanisms of $\omega-3$ LCPUFAs in normalizing the complications of COVID-19 in human beings.

\section{ACKNOWLEDGMENTS}

The authors would like to express their sincere gratitude to ICAR for providing funds to carry out the research work under ICAR-National Fellow Scheme. The authors acknowledge the Director, ICAR-Central Institute of Fisheries Technology (ICAR-CIFT), Cochin, Kerala, India for granting permission to publish the data acquired from the study.

\section{CONTRIBUTION OF AUTHORS}

Research concept- Dr. R Anandan

Research design- Dr. R Anandan, Dr. Suseela Mathew Supervision- Dr. R Anandan, Dr. Suseela Mathew, Dr. CN Ravishankar

Materials- M Rosemol Jacob, TS Fazil

Data collection- M Rosemol Jacob, TS Fazil

Data analysis and Interpretation- TS Fazil, Dr. R Anandan, Dr. Suseela Mathew

Literature search- M Rosemol Jacob, Dr. R Anandan, Dr. Suseela Mathew

Writing article- M Rosemol Jacob, Dr. R Anandan, Dr. Suseela Mathew

Critical review- Dr. Suseela Mathew, Dr. CN Ravishankar Article editing- M Rosemol Jacob, Dr. R Anandan

Final approval- Dr. Suseela Mathew, Dr. CN Ravishankar

\section{REFERENCES}

[1] Guertin MH, Robitaille K, Pelletier JF, Duchesne T, Julien $P$, et al. Effects of concentrated long-chain omega-3 polyunsaturated fatty acid supplementation before radical prostatectomy on prostate cancer proliferation, inflammation, and quality of life: study protocol for a phase $\mathrm{Ilb}$, randomized, double-blind, placebo-controlled trial. BMC cancer, 2018; 18(1): 110.

[2] Khodadadi E, Maroufi P, Khodadadi E, Esposito I, Ganbarov K, et al. Study of combining virtual screening and antiviral treatments of the Sars-CoV-2 (Covid-19). Microbial Pathogenesis, 2020; pp. 104241.

[3] Gutiérrez S, Svahn SL, Johansson ME. Effects of omega-3 fatty acids on immune cells. Int J Mol., 2019; 20(20): pp. 5028.

[4] Huang C, Wang Y, Li X, Ren L, Zhao J, et al. Clinical features of patients infected with 2019 novel coronavirus in Wuhan, China. The lancet, 2020; 395(10223): 497-506.

[5] Gurzell EA, Teague H, Duriancik D, Clinthorne J, Harris $M$, et al. Marine fish oils are not equivalent with respect to $B$-cell membrane organization and activation. J Nutr Biochem., 2015; 26(4): 369-77.

[6] Tseng YW, Wang PH, Lee HS, Liu BH, Mersmann HJ, et al. Regulation of the expression of angiotensinconverting enzyme 2 by polyunsaturated fatty acids in porcine adipocytes. J Anim Sci., 2010; 88(11): 3563-67.

[7] Zheng YY, Ma YT, Zhang JY, Xie X. COVID-19 and the cardiovascular system. Nature Rev Cardiol., 2020; 17(5): 259-60.

[8] Jang HY, Lim K, Lee SM, Park BH. Effects of n-3 PUFA on the CD4+ type 2 helper T-cell-mediated immune responses in Fat-1 mice. Mol Nutr Food Res., 2014; 58(2): 365-75.

[9] Liddle DM, Monk JM, Hutchinson AL, Ma DW, Robinson LE. CD8+ $T$ cell/adipocyte inflammatory cross talk and ensuing M1 macrophage polarization are reduced by fish-oil-derived $n-3$ polyunsaturated fatty acids, in part by a TNF- $\alpha$-dependent mechanism. J Nutr Biochem., 2020; 76: 108243.

[10]Gottrand F. Long-chain polyunsaturated fatty acids influence the immune system of infants. J Nutr., 2008; 138(9): 1807S-12S. 
[11]Swanson D, Block R, Mousa SA. Omega-3 fatty acids EPA and DHA: health benefits throughout life. Adv Nutr., 2012; 3(1): 1-7.

[12]Vanschoonbeek K, Wouters K, Van der Meijden PE, Van Gorp PJ, et al. Anticoagulant effect of dietary fish oil in hyperlipidemia: A study of hepatic gene expression in APOE2 knock-in mice. Arterioscler Thromb Vasc Biol., 2008; 28(11): 2023-59.

[13]Anandan R, Mathew S, Sankar TV, Nair PV. Protective effect of $n-3$ polyunsaturated fatty acids concentrate on isoproterenol-induced myocardial infarction in rats. Prostaglandins Leukot Essent Fatty Acids, 2007; 76(3): 153-58. 